Abbott, B.;...; Hosken, David John; Hough, J.;...; Munch, Jesper; Murray, P. G.;...; Ottaway, David John; Ottens, R. S.;...; Veitch, Peter John; ... et al.; LIGO Scientific Collaboration All-Sky LIGO Search for Periodic Gravitational Waves in the Early Fifth-Science-Run Data Physical Review Letters, 2009; 102(11):111102

(C2009 American Physical Society

http://link.aps.org/doi/10.1103/PhysRevLett.102.111102

\title{
PERMISSIONS
}

http://publish.aps.org/authors/transfer-of-copyright-agreement

"The author(s), and in the case of a Work Made For Hire, as defined in the U.S.

Copyright Act, 17 U.S.C.

$\S 101$, the employer named [below], shall have the following rights (the "Author Rights"):

$[\ldots]$

3. The right to use all or part of the Article, including the APS-prepared version without revision or modification, on the author(s)' web home page or employer's website and to make copies of all or part of the Article, including the APS-prepared version without revision or modification, for the author(s)' and/or the employer's use for educational or research purposes."

$10^{\text {th }}$ May 2013 


\section{All-Sky LIGO Search for Periodic Gravitational Waves in the Early Fifth-Science-Run Data}

B. P. Abbott,${ }^{17}$ R. Abbott,${ }^{17}$ R. Adhikari, ${ }^{17}$ P. Ajith,${ }^{2}$ B. Allen,,${ }^{2,57}$ G. Allen,${ }^{34}$ R. S. Amin, ${ }^{21}$ S. B. Anderson, ${ }^{17}$ W. G. Anderson, ${ }^{57}$ M. A. Arain, ${ }^{43}$ M. Araya, ${ }^{17}$ H. Armandula, ${ }^{17}$ P. Armor, ${ }^{57}$ Y. Aso, ${ }^{10}$ S. Aston, ${ }^{42}$ P. Aufmuth, ${ }^{16}$ C. Aulbert, ${ }^{2}$ S. Babak, ${ }^{1}$ P. Baker, ${ }^{24}$ S. Ballmer, ${ }^{17}$ H. Bantilan, ${ }^{8}$ B. C. Barish,${ }^{17}$ C. Barker, ${ }^{18}$ D. Barker, ${ }^{18}$ B. Barr, ${ }^{44}$ P. Barriga ${ }^{56}$ L. Barsotti, ${ }^{20}$ M. A. Barton, ${ }^{44}$ I. Bartos,${ }^{10}$ R. Bassiri,${ }^{44}$ M. Bastarrika,${ }^{44}$ B. Behnke, ${ }^{1}$ M. Benacquista ${ }^{38}$ J. Betzwieser, ${ }^{17}$ P. T. Beyersdorf,${ }^{30}$ I. A. Bilenko, ${ }^{25}$ G. Billingsley, ${ }^{17}$ R. Biswas,${ }^{57}$ E. Black, ${ }^{17}$ J. K. Blackburn, ${ }^{17}$ L. Blackburn, ${ }^{20}$ D. Blair,${ }^{56}$ B. Bland,${ }^{18}$ T. P. Bodiya,${ }^{20}$ L. Bogue,${ }^{19}$ R. Bork, ${ }^{17}$ V. Boschi, ${ }^{17}$ S. Bose,${ }^{58}$ P. R. Brady, ${ }^{57}$ V. B. Braginsky, ${ }^{25}$ J. E. Brau, ${ }^{50}$ M. Brinkmann, ${ }^{2}$ A. F. Brooks, ${ }^{17}$ D. A. Brown, ${ }^{35}$ G. Brunet,${ }^{20}$ A. Bullington, ${ }^{34}$ A. Buonanno, ${ }^{45}$ O. Burmeister, ${ }^{2}$ R. L. Byer, ${ }^{34}$ L. Cadonati, ${ }^{46}$ G. Cagnoli, ${ }^{44}$ J. B. Camp,${ }^{26}$ J. Cannizzo, ${ }^{26}$ K. C. Cannon, ${ }^{17}$ J. Cao, ${ }^{20}$ L. Cardenas, ${ }^{17}$ V. Cardoso, ${ }^{49}$ S. Caride, ${ }^{8}$ T. Casebolt,${ }^{34}$ G. Castaldi, ${ }^{53}$ S. Caudill, ${ }^{21}$ M. Cavaglià, ${ }^{49}$ C. Cepeda, ${ }^{17}$ E. Chalkley, ${ }^{44}$ P. Charlton, ${ }^{9}$ S. Chatterji,${ }^{17}$ S. Chelkowski, ${ }^{42}$ Y. Chen, ${ }^{1}$ N. Christensen, ${ }^{8}$ D. Clark, ${ }^{34}$ J. Clark, ${ }^{44}$ J. H. Clayton, ${ }^{57}$ T. Cokelaer, ${ }^{7}$ R. Conte, ${ }^{52}$ D. Cook, ${ }^{18}$ T. R. C. Corbitt, ${ }^{20}$ N. Cornish, ${ }^{24}$ D. C. Coyne, ${ }^{17}$ J. D. E. Creighton, ${ }^{57}$ T. D. Creighton, ${ }^{38}$ A. M. Cruise, ${ }^{42}$ A. Cumming, ${ }^{44}$ L. Cunningham, ${ }^{44}$ R. M. Cutler, K. Danzmann, ${ }^{2,16}$ B. Daudert, ${ }^{17}$ G. Davies, ${ }^{7}$ D. DeBra,${ }^{34}$ J. Degallaix, ${ }^{2}$ V. Dergachev, ${ }^{47}$ S. Desai,${ }^{36}$ R. DeSalvo, ${ }^{17}$ S. Dhurandhar,${ }^{15}$ M. Díaz,${ }^{38}$ J. Dickson, ${ }^{4}$ A. Dietz, ${ }^{7}$ F. Donovan, ${ }^{20}$ K. L. Dooley,${ }^{43}$ E. E. Doomes,${ }^{33}$ R. W. P. Drever,${ }^{5}$ I. Duke, ${ }^{20}$ J.-C. Dumas, ${ }^{56}$ J. Dwyer, ${ }^{10}$ C. Echols, ${ }^{17}$ M. Edgar, ${ }^{44}$ A. Effler, ${ }^{18}$ P. Ehrens,${ }^{17}$ G. Ely, ${ }^{8}$ E. Espinoza, ${ }^{17}$ T. Etzel,${ }^{17}$ M. Evans, ${ }^{20}$ T. Evans, ${ }^{19}$ S. Fairhurst, ${ }^{7}$ Y. Faltas, ${ }^{43}$ Y. Fan ${ }^{56}$ D. Fazi,${ }^{17}$ M. M. Fejer, ${ }^{34}$ L. S. Finn ${ }^{36}$ K. Flasch, ${ }^{57}$ S. Foley, ${ }^{20}$ C. Forrest, ${ }^{51}$ N. Fotopoulos, ${ }^{57}$ A. Franzen, ${ }^{16}$ Z. Frei, ${ }^{12}$ A. Freise, ${ }^{42}$ R. Frey,${ }^{50}$ T. T. Fricke, ${ }^{17,51}$ P. Fritschel,${ }^{20}$ V. V. Frolov, ${ }^{19}$ M. Fyffe, ${ }^{19}$ J. A. Garofoli, ${ }^{18}$ I. Gholami, ${ }^{1}$ J. A. Giaime, ${ }^{19,21}$ S. Giampanis, ${ }^{51}$ K. D. Giardina, ${ }^{19}$ K. Goda,${ }^{20}$ E. Goetz, ${ }^{47}$ L. M. Goggin, ${ }^{17}$ G. González, ${ }^{21}$

S. Gossler, ${ }^{2}$ R. Gouaty, ${ }^{21}$ A. Grant,${ }^{44}$ S. Gras,${ }^{56}$ C. Gray, ${ }^{18}$ M. Gray, ${ }^{4}$ R. J. S. Greenhalgh, ${ }^{29}$ A. M. Gretarsson, ${ }^{11}$ F. Grimaldi, ${ }^{20}$ R. Grosso, ${ }^{38}$ H. Grote, ${ }^{2}$ S. Grunewald, ${ }^{1}$ M. Guenther, ${ }^{18}$ E. K. Gustafson, ${ }^{17}$ R. Gustafson, ${ }^{47}$ B. Hage, ${ }^{16}$ J. M. Hallam, ${ }^{42}$ C. Hanna, ${ }^{21}$ J. Hanson, ${ }^{19}$ J. Harms,${ }^{2}$ G. M. Harry, ${ }^{20}$ E. D. Harstad,${ }^{50}$ E. Haughian, ${ }^{44}$ K. Hayama, ${ }^{38}$ T. Hayler, ${ }^{29}$ J. Heefner,${ }^{17}$ I. S. Heng,${ }^{44}$ A. Heptonstall, ${ }^{17}$ M. Hewitson, ${ }^{2}$ S. Hild,${ }^{42}$ E. Hirose, ${ }^{35}$ D. Hoak,${ }^{19}$ K. Holt, ${ }^{19}$ D. Hosken, ${ }^{41}$ J. Hough, ${ }^{44}$ S. H. Huttner, ${ }^{44}$ D. Ingram,,${ }^{18}$ M. Ito ${ }^{50}$ A. Ivanov, ${ }^{17}$ B. Johnson, ${ }^{18}$ W. W. Johnson, ${ }^{21}$ D. I. Jones,${ }^{54}$ G. Jones, ${ }^{7}$ R. Jones, ${ }^{44}$ L. Ju, ${ }^{56}$ P. Kalmus, ${ }^{10}$ V. Kalogera, ${ }^{28}$ S. Kamat, ${ }^{10}$ J. Kanner, ${ }^{45}$ D. Kasprzyk, ${ }^{42}$ E. Katsavounidis, ${ }^{20}$ K. Kawabe, ${ }^{18}$ S. Kawamura, ${ }^{27}$ F. Kawazoe, ${ }^{27}$ W. Kells, ${ }^{17}$ D. G. Keppel, ${ }^{17}$ A. Khalaidovski, ${ }^{2}$ F. Ya. Khalili, ${ }^{25}$ R. Khan, ${ }^{10}$ E. Khazanov ${ }^{14}$ P. King, ${ }^{17}$ J. S. Kissel, ${ }^{21}$ S. Klimenko, ${ }^{43}$ B. Kocsis, ${ }^{12}$ K. Kokeyama, ${ }^{27}$ V. Kondrashov, ${ }^{17}$ R. Kopparapu, ${ }^{36}$ S. Koranda, ${ }^{57}$ D. Kozak, ${ }^{17}$ I. Kozhevatov, ${ }^{14}$ B. Krishnan, ${ }^{1}$ P. Kwee, ${ }^{16}$ M. Landry, ${ }^{18}$ B. Lantz, ${ }^{34}$ A. Lazzarini, ${ }^{17}$ M. Lei, ${ }^{17}$ I. Leonor, ${ }^{50}$ C. Li ${ }^{6}$ H. Lin, ${ }^{43}$ P. E. Lindquist,${ }^{17}$ T. B. Littenberg, ${ }^{24}$ N. A. Lockerbie, ${ }^{55}$ D. Lodhia, ${ }^{42}$ M. Lormand,${ }^{19}$ P. Lu, ${ }^{34}$ M. Lubinski, ${ }^{18}$ A. Lucianetti, ${ }^{43}$ H. Lück, ${ }^{2,16}$ B. Machenschalk, ${ }^{1}$ M. MacInnis, ${ }^{20}$ M. Mageswaran, ${ }^{17}$ K. Mailand, ${ }^{17}$ I. Mandel, ${ }^{28}$ V. Mandic,${ }^{48}$ S. Márka, ${ }^{10,12}$ Z. Márka, ${ }^{10}$ A. Markosyan, ${ }^{34}$ J. Markowitz,${ }^{20}$ E. Maros, ${ }^{17}$ I. W. Martin, ${ }^{44}$ R. M. Martin, ${ }^{43}$ J. N. Marx, ${ }^{17}$ K. Mason, ${ }^{20}$ F. Matichard,${ }^{21}$ L. Matone, ${ }^{10}$ R. Matzner,${ }^{37}$ N. Mavalvala, ${ }^{20}$ R. McCarthy, ${ }^{18}$

D. E. McClelland, ${ }^{4}$ S. C. McGuire, ${ }^{33}$ M. McHugh, ${ }^{23}$ G. McIntyre, ${ }^{17}$ D. McKechan, ${ }^{7}$ K. McKenzie, ${ }^{4}$ M. Mehmet ${ }^{2}$

A. Melissinos, ${ }^{51}$ G. Mendell ${ }^{18}$ R. A. Mercer, ${ }^{43}$ S. Meshkov, ${ }^{17}$ C. J. Messenger, ${ }^{2}$ D. Meyers,${ }^{19}$ A. Miller, ${ }^{57}$ J. Miller, ${ }^{44}$ J. Minelli, ${ }^{36}$ S. Mitra,${ }^{15}$ V. P. Mitrofanov,${ }^{25}$ G. Mitselmakher,${ }^{43}$ R. Mittleman, ${ }^{20}$ O. Miyakawa,${ }^{17}$ B. Moe,${ }^{57}$ S. D. Mohanty ${ }^{38}$ G. Moreno, ${ }^{18}$ K. Mors, ${ }^{2}$ K. Mossavi, ${ }^{2}$ C. MowLowry, ${ }^{4}$ G. Mueller, ${ }^{43}$ D. Muhammad, ${ }^{19}$ S. Mukherjee, ${ }^{38}$ H. Mukhopadhyay, ${ }^{15}$ A. Mullavey, ${ }^{4}$ H. Müller-Ebhardt, ${ }^{2}$ J. Munch, ${ }^{41}$ P. G. Murray, ${ }^{44}$ E. Myers, ${ }^{18}$ J. Myers, ${ }^{18}$ T. Nash, ${ }^{17}$ J. Nelson, ${ }^{44}$ G. Newton, ${ }^{44}$ A. Nishizawa, ${ }^{27}$ K. Numata, ${ }^{26}$ E. Ochsner, ${ }^{45}$ J. O’Dell, ${ }^{29}$ G. Ogin, ${ }^{17}$ B. O’Reilly, ${ }^{19}$

R. O’Shaughnessy, ${ }^{36}$ D. J. Ottaway, ${ }^{41}$ R. S. Ottens, ${ }^{43}$ H. Overmier, ${ }^{19}$ B. J. Owen, ${ }^{36}$ Y. Pan, ${ }^{45}$ C. Pankow, ${ }^{43}$ M. A. Papa ${ }^{57}$ V. Parameshwaraiah, ${ }^{18}$ P. Patel,${ }^{17}$ M. Pedraza, ${ }^{17}$ S. Penn, ${ }^{13}$ A. Perraca, ${ }^{42}$ T. Petrie,${ }^{36}$ I. M. Pinto, ${ }^{53}$ M. Pitkin, ${ }^{44}$ H. J. Pletsch, ${ }^{2}$ M. V. Plissi, ${ }^{44}$ F. Postiglione, ${ }^{52}$ M. Principe,${ }^{53}$ R. Prix ${ }^{2}$ V. Quetschke, ${ }^{43}$ F. J. Raab, ${ }^{18}$ D. S. Rabeling, ${ }^{4}$

H. Radkins, ${ }^{18}$ P. Raffai, ${ }^{12}$ N. Rainer, ${ }^{2}$ M. Rakhmanov, ${ }^{38}$ M. Ramsunder, ${ }^{36}$ T. Reed, ${ }^{22}$ H. Rehbein, ${ }^{2}$ S. Reid, ${ }^{44}$

D. H. Reitze ${ }^{43}$ R. Riesen, ${ }^{19}$ K. Riles ${ }^{47}$ B. Rivera, ${ }^{18}$ N. A. Robertson, ${ }^{17,44}$ C. Robinson, ${ }^{7}$ E. L. Robinson, ${ }^{42}$ S. Roddy, ${ }^{19}$ A. M. Rogan, ${ }^{58}$ J. Rollins, ${ }^{10}$ J. D. Romano, ${ }^{38,7}$ J. H. Romie, ${ }^{19}$ S. Rowan, ${ }^{44}$ A. Rüdiger, ${ }^{2}$ L. Ruet, ${ }^{20}$ P. Russell, ${ }^{17}$ K. Ryan, ${ }^{18}$ S. Sakata, ${ }^{27}$ L. Sancho de la Jordana, ${ }^{40}$ V. Sandberg, ${ }^{18}$ V. Sannibale, ${ }^{17}$ L. Santamaria, ${ }^{1}$ S. Saraf,${ }^{31}$ P. Sarin, ${ }^{20}$ B. S. Sathyaprakash ${ }^{7}$ S. Sato, ${ }^{27}$ P. R. Saulson, ${ }^{35}$ R. Savage, ${ }^{18}$ P. Savov ${ }^{6}$ M. Scanlan, ${ }^{22}$ S. W. Schediwy, ${ }^{56}$ R. Schilling, ${ }^{2}$ R. Schnabel, ${ }^{2}$ R. Schofield, ${ }^{50}$ B. F. Schutz, ${ }^{1,7}$ P. Schwinberg, ${ }^{18}$ J. Scott, ${ }^{44}$ S. M. Scott, ${ }^{4}$ A. C. Searle, ${ }^{17}$ B. Sears, ${ }^{17}$ F. Seifert, ${ }^{2}$ D. Sellers, ${ }^{19}$ A. S. Sengupta, ${ }^{17}$ A. Sergeev, ${ }^{14}$ B. Shapiro, ${ }^{20}$ P. Shawhan, ${ }^{45}$ D. H. Shoemaker, ${ }^{20}$ A. Sibley, ${ }^{19}$ X. Siemens,${ }^{57}$ D. Sigg, ${ }^{18}$ S. Sinha, ${ }^{34}$ A. M. Sintes,${ }^{40,1}$ B. J. J. Slagmolen, ${ }^{4}$ J. Slutsky, ${ }^{21}$ J. R. Smith, ${ }^{35}$ M. R. Smith, ${ }^{17}$ N. D. Smith, ${ }^{20}$ 
K. Somiya, ${ }^{1,2}$ B. Sorazu, ${ }^{44}$ L. C. Stein, ${ }^{20}$ K. A. Strain,${ }^{44}$ A. Stuver,${ }^{19}$ T. Z. Summerscales, ${ }^{3}$ K.-X. Sun,${ }^{34}$ M. Sung, ${ }^{21}$ P. J. Sutton, ${ }^{7}$ H. Takahashi, ${ }^{1}$ D. B. Tanner, ${ }^{43}$ R. Taylor, ${ }^{17}$ R. Taylor,${ }^{44}$ J. Thacker, ${ }^{19}$ K. A. Thorne,${ }^{36}$ K. S. Thorne,${ }^{6}$ A. Thüring, ${ }^{16} \mathrm{~K}$. V. Tokmakov, ${ }^{44} \mathrm{C}$. Torres,${ }^{19} \mathrm{C}$. Torrie, ${ }^{44} \mathrm{G}$. Traylor, ${ }^{19} \mathrm{M}$. Trias,${ }^{40}$ D. Ugolini,${ }^{39} \mathrm{~K}$. Urbanek,${ }^{34}$ H. Vahlbruch, ${ }^{16}$ C. Van Den Broeck, ${ }^{7}$ M. V. van der Sluys, ${ }^{28}$ A. A. van Veggel,${ }^{44}$ S. Vass,${ }^{17}$ R. Vaulin, ${ }^{57}$ A. Vecchio ${ }^{42}$ J. D. Veitch, ${ }^{42}$ P. Veitch, ${ }^{41}$ A. Villar, ${ }^{17}$ C. Vorvick, ${ }^{18}$ S. P. Vyachanin, ${ }^{25}$ S. J. Waldman, ${ }^{17}$ L. Wallace, ${ }^{17}$ H. Ward, ${ }^{44}$ R. L. Ward ${ }^{17}$ M. Weinert, ${ }^{2}$ A. J. Weinstein, ${ }^{17}$ R. Weiss, ${ }^{20}$ L. Wen, ${ }^{6}$ S. Wen,${ }^{21}$ K. Wette, ${ }^{4}$ J. T. Whelan, ${ }^{1}$ S. E. Whitcomb, ${ }^{17}$ B. F. Whiting, ${ }^{43}$ C. Wilkinson, ${ }^{18}$ P. A. Willems, ${ }^{17}$ H. R. Williams,${ }^{36}$ L. Williams,${ }^{43}$ B. Willke, ${ }^{2,16}$ I. Wilmut, ${ }^{29}$ W. Winkler, ${ }^{2}$ C. C. Wipf,${ }^{20}$ A. G. Wiseman, ${ }^{57}$ G. Woan,${ }^{44}$ R. Wooley, ${ }^{19}$ J. Worden, ${ }^{18}$ W. Wu ${ }^{43}$ I. Yakushin,,${ }^{19}$ H. Yamamoto, ${ }^{17}$ Z. Yan, ${ }^{56}$ S. Yoshida, ${ }^{32}$ M. Zanolin, ${ }^{11}$ J. Zhang, ${ }^{47}$ L. Zhang, ${ }^{17}$ C. Zhao, ${ }^{56}$ N. Zotov, ${ }^{22}$ M. E. Zucker, ${ }^{20}$ H. zur Mühlen, ${ }^{16}$ and J. Zweizig ${ }^{17}$

(LIGO Scientific Collaboration)* $^{*}$

\footnotetext{
${ }^{1}$ Albert-Einstein-Institut, Max-Planck-Institut für Gravitationsphysik, D-14476 Golm, Germany ${ }^{2}$ Albert-Einstein-Institut, Max-Planck-Institut für Gravitationsphysik, D-30167 Hannover, Germany

${ }^{3}$ Andrews University, Berrien Springs, Michigan 49104, USA

${ }^{4}$ Australian National University, Canberra, 0200, Australia

${ }^{5}$ California Institute of Technology, Pasadena, California 91125, USA

${ }^{6}$ Caltech-CaRT, Pasadena, California 91125, USA

${ }^{7}$ Cardiff University, Cardiff, CF24 3AA, United Kingdom

${ }^{8}$ Carleton College, Northfield, Minnesota 55057, USA

${ }^{9}$ Charles Sturt University, Wagga Wagga, NSW 2678, Australia

${ }^{10}$ Columbia University, New York, New York 10027, USA

${ }^{11}$ Embry-Riddle Aeronautical University, Prescott, Arizona 86301, USA

${ }^{12}$ Eötvös University, ELTE 1053 Budapest, Hungary

${ }^{13}$ Hobart and William Smith Colleges, Geneva, New York 14456, USA

${ }^{14}$ Institute of Applied Physics, Nizhny Novgorod, 603950, Russia

${ }^{15}$ Inter-University Centre for Astronomy and Astrophysics, Pune-411007, India

${ }^{16}$ Leibniz, Universität Hannover, D-30167 Hannover, Germany

${ }^{17}$ LIGO-California Institute of Technology, Pasadena, California 91125, USA

${ }^{18}$ LIGO-Hanford Observatory, Richland, Washington 99352, USA

${ }^{19}$ LIGO-Livingston Observatory, Livingston, Louisiana 70754, USA

${ }^{20}$ LIGO-Massachusetts Institute of Technology, Cambridge, Massachusetts 02139, USA

${ }^{21}$ Louisiana State University, Baton Rouge, Louisiana 70803, USA

${ }^{22}$ Louisiana Tech University, Ruston, Louisiana 71272, USA

${ }^{23}$ Loyola University, New Orleans, Louisiana 70118, USA

${ }^{24}$ Montana State University, Bozeman, Montana 59717, USA

${ }^{25}$ Moscow State University, Moscow, 119992, Russia

${ }^{26}$ NASA/Goddard Space Flight Center, Greenbelt, Maryland 20771, USA

${ }^{27}$ National Astronomical Observatory of Japan, Tokyo 181-8588, Japan

${ }^{28}$ Northwestern University, Evanston, Illinois 60208, USA

${ }^{29}$ Rutherford Appleton Laboratory, Chilton, Didcot, Oxon OX11 OQX, United Kingdom

${ }^{30}$ San Jose State University, San Jose, California 95192, USA

${ }^{31}$ Sonoma State University, Rohnert Park, California 94928, USA

${ }^{32}$ Southeastern Louisiana University, Hammond, Louisiana 70402, USA

${ }^{33}$ Southern University and A\&M College, Baton Rouge, Louisiana 70813, USA

${ }^{34}$ Stanford University, Stanford, California 94305, USA

${ }^{35}$ Syracuse University, Syracuse, New York 13244, USA

${ }^{36}$ The Pennsylvania State University, University Park, Pennsylvania 16802, USA

${ }^{37}$ The University of Texas at Austin, Austin, Texas 78712, USA

${ }^{38}$ The University of Texas at Brownsville and Texas Southmost College, Brownsville, Texas 78520, USA

${ }^{39}$ Trinity University, San Antonio, Texas 78212, USA

${ }^{40}$ Universitat de les Illes Balears, E-07122 Palma de Mallorca, Spain

${ }^{41}$ University of Adelaide, Adelaide, SA 5005, Australia

${ }^{42}$ University of Birmingham, Birmingham, B15 2TT, United Kingdom

${ }^{43}$ University of Florida, Gainesville, Florida 32611, USA

${ }^{44}$ University of Glasgow, Glasgow, G12 8QQ, United Kingdom

${ }^{45}$ University of Maryland, College Park, Maryland 20742, USA
} 


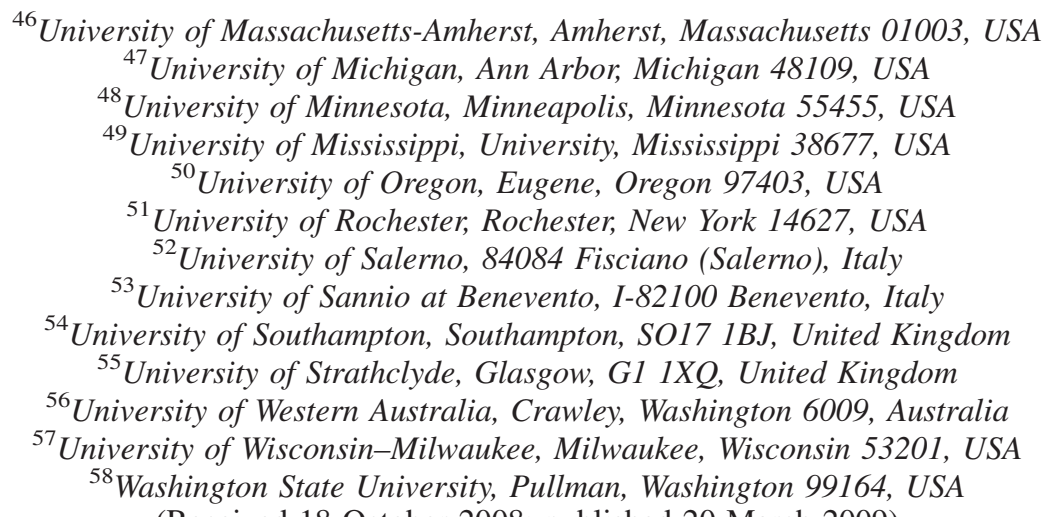

(Received 18 October 2008; published 20 March 2009)

\begin{abstract}
We report on an all-sky search with the LIGO detectors for periodic gravitational waves in the frequency range $50-1100 \mathrm{~Hz}$ and with the frequency's time derivative in the range $-5 \times 10^{-9}-0 \mathrm{~Hz} \mathrm{~s}^{-1}$. Data from the first eight months of the fifth LIGO science run (S5) have been used in this search, which is based on a semicoherent method (PowerFlux) of summing strain power. Observing no evidence of periodic gravitational radiation, we report $95 \%$ confidence-level upper limits on radiation emitted by any unknown isolated rotating neutron stars within the search range. Strain limits below $10^{-24}$ are obtained over a $200-\mathrm{Hz}$ band, and the sensitivity improvement over previous searches increases the spatial volume sampled by an average factor of about 100 over the entire search band. For a neutron star with nominal equatorial ellipticity of $10^{-6}$, the search is sensitive to distances as great as $500 \mathrm{pc}$.
\end{abstract}

DOI: 10.1103/PhysRevLett.102.111102

Introduction.-We have carried out an all-sky search with the Laser Interferometer Gravitational-Wave Observatory (LIGO) detectors [1,2] for periodic gravitational waves, using data from the first eight months of LIGO's fifth science run (S5). We have searched over the frequency range $50-1100 \mathrm{~Hz}$, allowing for a frequency time derivative in the range $-5 \times 10^{-9}-0 \mathrm{~Hz} \mathrm{~s}^{-1}$. These parameter ranges correspond to a minimum spin-down time scale $f /|4 \dot{f}|$ (the gravitational-wave spin-down age) of $80 \mathrm{yr}$ for a source emitting at $50 \mathrm{~Hz}$ and $1750 \mathrm{yr}$ for a source at $1100 \mathrm{~Hz}$. Rotating neutron stars in our Galaxy are the prime target. At signal frequencies near $100 \mathrm{~Hz}$ we obtain strain sensitivities below $10^{-24}$, a strain at which one might optimistically expect to see the strongest signal from a previously unknown neutron star according to a generic argument originally made by Blandford [3], and extended in our previous search for such objects in S2 data [4]. A recent refinement of the argument [5] gives less optimistic estimates, but these too are surpassed by the experimental results presented here.

Using data from earlier science runs, the LIGO Scientific Collaboration has previously reported on allsky searches for unknown rotating neutron stars (henceforth designated as "pulsars"). These searches have been performed using a short-period coherent search in the $160.0-728.8 \mathrm{~Hz}$ frequency range [4], and using a longperiod semicoherent search in the $200-400 \mathrm{~Hz}$ frequency range in the S2 data [6] and the $50-1000 \mathrm{~Hz}$ range in the S4 data [7]. Einstein@Home, a distributed home computing effort [8], has also been running searches using a co-
PACS numbers: 04.80.Nn, 07.05.Kf, 95.55.Ym, 97.60.Gb

herent first stage, followed by a simple coincidence stage, for which S3 and S4 results have been released $[9,10]$.

The data collected in the S5 data run were more sensitive than in previous data runs, and the amount of data used here is an increase by a factor of 8 over that reported from the S4 data run [7], resulting in upper limits on periodic gravitational waves about a factor of 3-6 lower than those from the S4 data, depending on source frequency. This improvement gives an increase in sampled galactic volume by about a factor of 100, depending on the assumed source frequency and spin-down. At a signal frequency of $1100 \mathrm{~Hz}$ we achieve sensitivity to neutron stars of equatorial ellipticity $\epsilon \sim 10^{-6}$ at distances up to $500 \mathrm{pc}$ (see [7] for relations). This ellipticity is at the upper end of the range thought to be sustainable by conventional neutron stars [11] and orders of magnitude below the maximum sustainable by a strange quark star [12]. The number of undiscovered, electromagnetically quiet neutron stars within $500 \mathrm{pc}$ can be estimated to be $O\left(10^{4}-10^{5}\right)$ from the neutron star birth rate [13], although it is likely that only a tiny fraction would both be rotating fast enough to be accessible to LIGO [14] and remain in the local volume over the age of the Galaxy [15]. Only $\sim 25$ radio or x-ray pulsars have been discovered so far within that volume [16].

LIGO detectors and the S5 science run.-The LIGO detector network consists of a 4-km interferometer in Livingston, Louisiana, (L1), and two interferometers in Hanford, Washington, one $4 \mathrm{~km}$ and the other $2 \mathrm{~km}$ (H1 and $\mathrm{H} 2$ ). 
The data analyzed in this Letter were produced in the first eight months of LIGO's fifth science run. This run started at 16:00 UTC on November 4, 2005, at the LIGOHanford Observatory and at 16:00 UTC on November 14, 2005, at the LIGO-Livingston Observatory; the run ended at 00:00 UTC on October 1, 2007. During this run, all three LIGO detectors had displacement spectral amplitudes very near their design goals of $1.1 \times 10^{-19} \mathrm{~m} \mathrm{~Hz}^{-1 / 2}$ [17] in their most sensitive frequency band near $150 \mathrm{~Hz}$. (In terms of gravitational-wave strain, the $\mathrm{H} 2$ interferometer was roughly a factor of 2 less sensitive than the other two; its data were not used in this search.) Because of a variety of technical computing issues, a search over the full S5 data set ( 2 yr) using the current PowerFlux program described below is impractical. Extensive revamping of the program is underway; results of the final ensuing search will be reported in the future.

The data were acquired and digitized at a rate of $16384 \mathrm{~Hz}$. Data acquisition was periodically interrupted by disturbances such as seismic transients (natural or anthropogenic), reducing the net running time of the interferometers. In addition, there were 1-2 week commissioning breaks to repair equipment and address newly identified noise sources. The resulting duty factors for the interferometers were approximately $69 \%$ for $\mathrm{H} 1,77 \%$ for $\mathrm{H} 2$, and $57 \%$ for $\mathrm{L} 1$ during the first eight months. A nearby construction project degraded the L1 duty factor significantly during this early period of the S5 run. By the end of the S5 run, the cumulative duty factors had improved to $78 \%$ for $\mathrm{H} 1,79 \%$ for $\mathrm{H} 2$, and $66 \%$ for $\mathrm{L} 1$. For this search, approximately $4077 \mathrm{~h}$ of $\mathrm{H} 1$ data and $3070 \mathrm{~h}$ of L1 data were used, where each data segment used was required to contain at least $30 \mathrm{~min}$ of continuous interferometer operation.

Signal waveforms. - The general form of a gravitationalwave signal is described in terms of two orthogonal transverse polarizations defined to be "+" with waveform $h_{+}(t)$ and "X" with waveform $h_{\times}(t)$, for which separate and time-dependent antenna pattern factors $F_{+}$and $F_{\times}$ apply, which depend on a polarization angle $\psi$ [18]. For periodic gravitational waves, which in general are elliptically polarized, the individual components $h_{+, \times}$have the form $h_{+}(t)=A_{+} \cos \Phi(t)$ and $h_{\times}(t)=A_{\times} \sin \Phi(t)$, where $A_{+}$and $A_{\times}$are the amplitudes of the two polarizations, and $\Phi(t)$ is the phase of the signal at the detector. For the semicoherent method used in this search, only the instantaneous signal frequency in the detector reference frame, $2 \pi f(t)=d \Phi(t) / d t$, needs to be calculated. For an isolated, precession-free, rigidly rotating neutron star the quadrupolar amplitudes $A_{+}$and $A_{\times}$are related to wave amplitude, $h_{0}$, by $A_{+}=h_{0}\left[\left(1+\cos ^{2} \iota\right) / 2\right]$ and $A_{\times}=$ $h_{0} \cos \iota$, where $\iota$ describes the inclination angle of the star's spin axis with respect to the line of sight. For such a star, the signal wave frequency $f$ is twice the rotation frequency $f_{r}$.
The detector reference frame frequency $f(t)$ can, to a very good approximation, be related to the frequency $\hat{f}(t)$ in the solar system barycenter (SSB) frame by [6] $f(t)-$ $\hat{f}(t) \simeq \hat{f}(t) \frac{\mathbf{v}(t) \cdot \mathbf{n}}{c}$, where $\mathbf{v}(t)$ is the detector's velocity with respect to the SSB frame, and $\mathbf{n}$ is the unit vector pointing from the detector toward the sky location of the source [6].

Analysis method.-The PowerFlux method used in this analysis is described in detail elsewhere [7] and is a variation upon the StackSlide method [19]. Here we summarize briefly its main features.

A strain power estimator is derived from summing measures of strain power from many short, 50\% overlap, Hannwindowed Fourier transforms (SFTs) that have been created from 30-min intervals of calibrated strain data. In searching a narrow frequency range $(0.5 \mathrm{mHz}$ spacing) for an assumed source sky location, explicit corrections are made for Doppler modulations of the apparent source frequency. These modulations are due to the Earth's rotation and its orbital motion around the SSB, and the frequency's time derivative $\dot{f}$ intrinsic to the source. Corrections are also applied for antenna pattern modulation, assuming five different polarizations: four linear polarizations separated by $\pi / 8$ in polarization angle and circular polarization. When summing, the variability of the noise is taken into account with a SFT-dependent weight proportional to the expected inverse variance of the background noise power (see $[7,20]$ for detailed formulas).

The search range for initial frequency $\hat{f}_{0}$ values is 50 $1100 \mathrm{~Hz}$ with a uniform grid spacing equal to the size of a SFT frequency bin $[1 /(30 \mathrm{~min})]$. The range of $\dot{f}$ values searched is $-5 \times 10^{-9}-0 \mathrm{~Hz} \mathrm{~s}^{-1}$ with a spacing of $5 \times$ $10^{-10} \mathrm{~Hz} \mathrm{~s}^{-1}$, since isolated rotating neutron stars are generally expected to spin down with time. As discussed in our previous reports $[4,6,7]$, the number of sky points that must be searched grows quadratically with the frequency $\hat{f}_{0}$, ranging here from about 5000 at $50 \mathrm{~Hz}$ to about $2.4 \times 10^{6}$ at $1100 \mathrm{~Hz}$. The sky grid used here is isotropic and covers the entire sky.

As described in [7], upper limits are computed from the highest-strain signal estimator over the entire sky for each $0.25-\mathrm{Hz}$ band. Upper limits calculated in this method are strict frequentist limits on linear and circular polarization in small patches on the sky, with the limits quoted here being the highest limits in each $0.25-\mathrm{Hz}$ band over broad regions of the sky. These are interpreted as limits on worstcase (linear polarization) and best-case (circular polarization) orientations of rotating neutron stars. Since the eight months of data analyzed here cover a large span of the Earth's orbit, providing substantial Doppler modulation of source frequency, contamination from stationary instrumental lines is much reduced from earlier and shorter data runs. A total of only $0.6 \%$ of the search volume in sky location and spin-down had to be excluded from the upper-limit analysis because of Doppler stationarity. 
The primary changes in the PowerFlux algorithm used in this search concern follow up of outlier candidates. (The general method for setting upper limits is identical to that used in the S4 search [7].) Here we summarize the followup method used. Single-interferometer searches are carried out separately for the $\mathrm{H} 1$ and $\mathrm{L} 1$ interferometers, leading to the upper limits on strain shown in Fig. 1 and discussed below. During determination of the maximum upper limit per sky region, per frequency band, and per spin-down step, a "domain map" is constructed of local signal-tonoise ratio (SNR) maxima, with the domains ordered by maximum grid point SNR and clustered if close in direction and frequency. The 1000 domains with the highest maximum SNR are then reanalyzed to obtain improved estimates of the associated candidate parameters, using a modified gradient search with a matched filter to maximize SNR with respect to source frequency, spin-down, sky location, polarization angle $\psi$, and inclination angle $\iota$ [20]. This maximization step samples frequency and spin-down much more finely than in the initial search.

When all sky regions and all spin-downs have been searched for a given $0.25 \mathrm{~Hz}$ band for both the $\mathrm{H} 1$ and L1 interferometers, the search pipeline outputs are compared, and the following criteria are used to define candidates for follow-up analysis. The $\mathrm{H} 1$ and $\mathrm{L} 1$ candidates must each have a SNR value greater than 6.25 , and they must agree in frequency to within $1 / 180 \mathrm{~Hz}=5.56 \mathrm{mHz}$, in spin-down to within $4 \times 10^{-10} \mathrm{~Hz} \mathrm{~s}^{-1}$, and in sky location to within $0.14 \mathrm{rad}$. These conservative choices have been guided by simulated single-interferometer pulsar in-

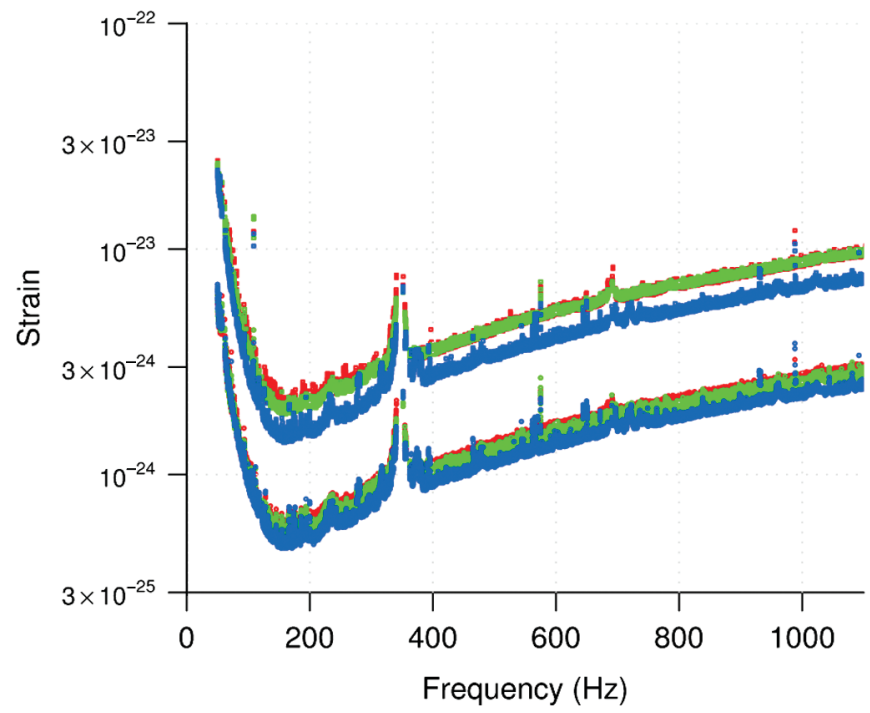

FIG. 1 (color). Minimum (H1 or L1) upper limits (95\% C.L.) on pulsar gravitational-wave amplitude $h_{0}$ for the equatorial (red), intermediate (green), and polar (blue) declination bands for best-case (lower curves) and worst-case (upper curves) pulsar orientations. Shown are all the minimum limits for each of the 11 spin-down values from $-5 \times 10^{-9} \mathrm{~Hz} \mathrm{~s}^{-1}$ to zero in steps of $5 \times 10^{-10} \mathrm{~Hz} \mathrm{~s}^{-1}$. jections. Coincidence candidates within $0.1 \mathrm{~Hz}$ of one another are grouped together, since most candidates arise from detector spectral artifacts that become apparent upon manual investigation.

Candidates passing these criteria are subjected to a computationally intensive follow-up analysis that reproduces the all-sky PowerFlux search in a $0.25 \mathrm{~Hz}$ band around each candidate, this time using the (incoherently) combined strain powers from both interferometers. Sky maps of strain and SNR are created and examined manually for each individual interferometer and for the combined interferometers. Spectral estimates from noise decomposition are also examined to identify possible artifacts leading to the coincident outliers.

Results.-Figure 1 shows the lower of the $\mathrm{H} 1$ and L1 95\% confidence-level upper limits on pulsar gravitationalwave amplitude $h_{0}$ for worst-case and best-case pulsar orientations for different declination bands (each with different run-averaged antenna pattern sensitivity). As in the S4 analysis, narrow frequency bands centered on the harmonics of the $60-\mathrm{Hz}$ power mains, along with bands characterized by non-Gaussian noise, have been excluded from the displayed limits. Numerical values for frequencies and limits displayed in these figures can be obtained separately [21]. Systematic uncertainties on these values are dominated by calibration uncertainty at the $\sim 10 \%$ level.

All outliers were checked for coincidence between H1 and L1 interferometers, as described above. In most cases single-interferometer spectral artifacts were readily found upon initial inspection, most of which had known instrumental or environmental sources, such as mechanical resonances ("violin modes") of the wires supporting interferometer mirrors, and power-mains harmonics of $60 \mathrm{~Hz}$. Other outliers were tracked down to previously unknown electromagnetic disturbances. For six coincidence candidates, all at frequencies above $850 \mathrm{~Hz}$, no instrumental spectral artifacts were apparent.

None of these six candidates were confirmed, however, as a detection of a constant-amplitude, constant-spin-down periodic source of gravitational radiation. In each case, we found that the combined H1-L1 SNR did not increase by more than 0.6 (0.4) units over the minimum (maximum) of the single-interferometer SNR's, with four candidates showing a decrease for combined SNR. To understand the expectation for a true signal, we carried out a posteriori software signal injections, which indicated that combined SNR should typically show an increase over minimum SNR by more than 2.0 units for a singleinterferometer SNR threshold of 6.25. Hence we conservatively veto all candidates with a SNR increase less than 1 unit. In addition, manual exploration of these candidates was carried out, using larger portions of the S5 run's data, to determine whether SNR increased with additional data, and with subsets of the original eight-month data, to de- 
termine whether a transient astrophysical source could explain the candidate. None of these explorations proved fruitful. It should be noted that this opportunistic search for a coincidence detection does not affect the upper limits shown in Fig. 1. Also, because of large variation in average antenna pattern sensitivity with respect to source declination, the high-SNR coincidence candidates do not, in general, coincide with the outliers that define those upper limits.

We also note that multi-interferometer injections indicate that for signal frequencies above $850 \mathrm{~Hz}$, the coincidence requirements in frequency and sky location could be tightened by a factor of 5 to $1 \mathrm{mHz}$ and by a factor of 7 to $0.02 \mathrm{rad}$, respectively, with only a slight reduction in efficiency for true signals. None of the six candidates mentioned above satisfy these tighter criteria.

In summary, we have set strict, all-sky frequentist upper limits on the strength of continuous-wave gravitational radiation of linear and circular polarization, corresponding to least favorable and most favorable pulsar orientations, respectively. Follow-up analysis of coincidence candidates with SNR $>6.25$ did not yield a detection. The limits on detected strain can be translated into limits on equatorial ellipticity as small as $10^{-6}$ for unknown neutron stars as far away as $500 \mathrm{pc}$. This ellipticity is at the upper end of the range thought to be sustainable by conventional neutron stars and orders of magnitude below the maximum sustainable by a strange quark star. Our limits on strain amplitude lie below (are more stringent than) the highest values in recent population simulations [5]. In the context of these models, our observational results imply that the galactic population of neutron stars spinning down primarily due to gravitational radiation approximately satisfies one or more of the following conditions: birth rate less than one per $30 \mathrm{yr}$, typical ellipticity less than $10^{-6}$, or typical spin period greater than $10 \mathrm{~ms}$.

We thank Deepto Chakrabarty and David Kaplan for useful discussions. The authors gratefully acknowledge the support of the U.S. National Science Foundation for the construction and operation of the LIGO Laboratory and the Science and Technology Facilities Council of the U.K., the Max Planck Society, and the State of Niedersachsen, Germany, for support of the construction and operation of the GEO600 detector. The authors also gratefully acknowledge the support of the research by these agencies and by the Australian Research Council, the Council of Scientific and Industrial Research of India, the Istituto Nazionale di Fisica Nucleare of Italy, the Spanish Ministerio de Educación y Ciencia, the Conselleria d'Economia Hisenda i Innovació of the Govern de les
Illes Balears, the Royal Society, the Scottish Funding Council, the Scottish Universities Physics Alliance, The National Aeronautics and Space Administration, the Carnegie Trust, the Leverhulme Trust, the David and Lucile Packard Foundation, the Research Corporation, and the Alfred P. Sloan Foundation.

*http://www.ligo.org

[1] A. Abramovici et al., Science 256, 325 (1992).

[2] B. Barish and R. Weiss, Phys. Today 52, No. 10, 44 (1999).

[3] R. Blandford (unpublished)

[4] B. Abbott et al. (LIGO Scientific Collaboration), Phys. Rev. D 76, 082001 (2007).

[5] B. Knispel and B. Allen, Phys. Rev. D 78, 044031 (2008).

[6] B. Abbott et al. (LIGO Scientific Collaboration), Phys. Rev. D 72, 102004 (2005).

[7] B. Abbott et al. (LIGO Scientific Collaboration), Phys. Rev. D 77, 022001 (2008).

[8] The Einstein@Home project is built upon the Berkeley Open Infrastructure for Network Computing (BOINC) architecture described at http://boinc.berkeley.edu/.

[9] S3 results from the distributed computing project Einstein@Home can be found at http://einstein.phys. uwm.edu/.

[10] B. Abbott et al. (LIGO Scientific Collaboration), Phys. Rev. D 79, 022001 (2009).

[11] G. Ushomirksy, C. Cutler, and L. Bildsten, Mon. Not. R. Astron. Soc. 319, 902 (2000).

[12] B. Owen, Phys. Rev. Lett. 95, 211101 (2005).

[13] R. Narayan, Astrophys. J. 319, 162 (1987).

[14] D. Lorimer, Living Rev. Relativity 8, 7 (2005), http:// relativity.livingreviews.org/Articles/lrr-2008-8/.

[15] J. M. Cordes and D. F. Chernoff, Astrophys. J. 505, 315 (1998).

[16] R. N. Manchester, G. B. Hobbs, A. Teoh, and M. Hobbs, Astron. J. 129, 1993 (2005); see also http://www.atnf. csiro.au/research/pulsar/psrcat/.

[17] B. Abbott et al. (LIGO Scientific Collaboration), arXiv:0711.3041.

[18] P. Jaranowski, A. Królak, and B. F. Schutz, Phys. Rev. D 58, 063001 (1998).

[19] P. Brady and T. Creighton, Phys. Rev. D 61, 082001 (2000).

[20] V. Dergachev, LIGO technical document LIGO-T050186, 2005, available in http://admdbsrv.ligo.caltech.edu/dcc/.

[21] See EPAPS Document No. E-PRLTAO-102-017912 for numerical values of upper limits derived for the $\mathrm{H} 1$ and L1 interferometers in $0.25-\mathrm{Hz}$ bands in the range $50-1000 \mathrm{~Hz}$. For more information on EPAPS, see http://www.aip.org/ pubservs/epaps.html. 